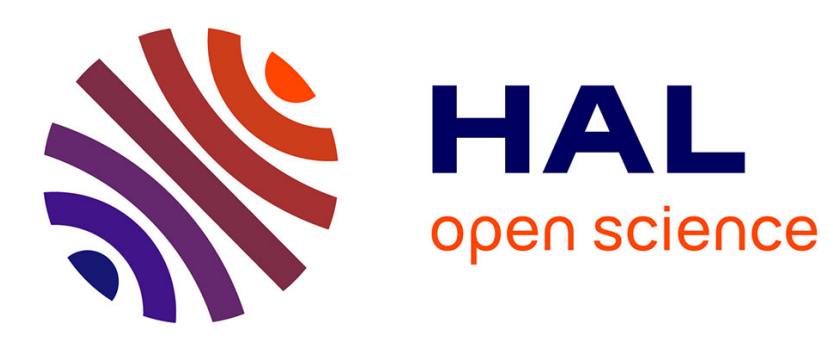

\title{
Blind identification of occurrence of multi-modality in laser-feedback-based self-mixing sensor
} Muhammad Usman, Usman Zabit, Olivier D Bernal, Gulistan Raja

\section{To cite this version:}

Muhammad Usman, Usman Zabit, Olivier D Bernal, Gulistan Raja. Blind identification of occurrence of multi-modality in laser-feedback-based self-mixing sensor. Chinese Optics Letters, 2020, 18 (1), pp.011201. 10.3788/COL202018.011201 . hal-03209572

\section{HAL Id: hal-03209572 \\ https://hal.laas.fr/hal-03209572}

Submitted on 27 Apr 2021

HAL is a multi-disciplinary open access archive for the deposit and dissemination of scientific research documents, whether they are published or not. The documents may come from teaching and research institutions in France or abroad, or from public or private research centers.
L'archive ouverte pluridisciplinaire HAL, est destinée au dépôt et à la diffusion de documents scientifiques de niveau recherche, publiés ou non, émanant des établissements d'enseignement et de recherche français ou étrangers, des laboratoires publics ou privés. 
(C) 2020 Optical Society of America]. One print or electronic copy may be made for personal use only. Systematic reproduction and distribution, duplication of any material in this paper for a fee or for commercial purposes, or modifications of the content of this paper are prohibited

\title{
Blind Identification of occurrence of multi-modality in laser feedback based self-mixing sensor
}

\author{
Muhammad Usman ${ }^{1}$, Usman Zabit ${ }^{2 *}$, Olivier D. Bernal ${ }^{3}$, and Gulistan Raja ${ }^{4}$ \\ ${ }^{1}$ Namal Institute, Mianwali, 42250, Pakistan \\ ${ }^{2}$ National University of Sciences \& Technology (NUST), Islamabad, 44000, Pakistan \\ ${ }^{3}$ Univ de Toulouse, INP, LAAS-CNRS, Toulouse F-31400, France \\ ${ }^{4}$ University of Engineering \& Technology, Taxila, 47080, Pakistan \\ * Corresponding author: usman.zabit@seecs.nust.edu.pk
}

\begin{abstract}
Self-mixing interferometry (SMI) is an attractive sensing scheme which typically relies on mono-modal operation of employed laser diode. However, change in laser modality can occur due to change in operating conditions. So, detection of occurrence of multi-modality in SMI signals is necessary to avoid erroneous metric measurements. Typically, processing of multi-modal SMI signals is a difficult task due to the diverse and complex nature of such signals. However, the proposed techniques can significantly ease this task by identifying the modal state of SMI signals with $100 \%$ success rate, so that interferometric fringes can be correctly interpreted for metric sensing applications.
\end{abstract}

\section{doi: $10.3788 /$ COL202018.011201}

SELF-MIXING (SM) or optical feedback (OF) interferometry ${ }^{[1,2]}$ is actively researched for vibration, angle ${ }^{[3]}$, frequency ${ }^{[4]}$, size ${ }^{[5]}$, range-finding ${ }^{[6]}$, topographical [7], and seismic applications ${ }^{[8]}$ due to the simple, low-cost, and miniaturized nature of SM sensors. In order to design low-cost SM sensors, usually commercial off the shelf (COTS) laser diodes (LD) are preferred. However, due to $\mathrm{OF}$ inside the active laser cavity, such low-cost monomodal LDs are prone to mode switching (as a function of operating conditions [9-11]) resulting in multi-modal SM signals in which more than one laser mode undergoes SM. As a consequence, each interferometric fringe can no more be assumed to correspond to a remote displacement of $\lambda / 2$ (where $\lambda$ is the wavelength of LD), because in case of bimodal or tri-modal SM, an individual SM fringe does not correspond to a displacement of $\lambda / 2$ anymore (see Fig.1) ${ }^{\text {[9] }}$. Multi-modal SM has been used to potentially increase measurement resolution [10] as well as to measure free spectral range of the laser [14]. However, any unidentified switching of a mono-modal laser sensor to multi-modal operation can cause any unidentified severe measurement error due to incorrect fringe interpretation.

The objective of this Letter is to robustly identify the occurrence of multi-modal SM signals so that an alert can be raised to appropriately interpret SM fringe-count and/or SM operating conditions can be changed (e.g. by changing LD current ${ }^{[12]}$ or amount of $\mathrm{OF}^{[13]}$ ) to revert back to monomodal SM operation [14,15] for which algorithms exist yielding high accuracy measurements ${ }^{[16-20]}$.

SM based multi-modality is reported to occur due to variation in parameters such as $\mathrm{LD}$-to-target distance ${ }^{[21,22]}$, temperature ${ }^{[9]}$, or LD current ${ }^{[10,12]}$. Measurement of laser emission spectra confirmed existence of multiple laser modes undergoing SM signal ${ }^{[9,12,23]}$ for different laser sources such as Fabry-Perot LD [21], quantum cascade laser ${ }^{[23]}$, and VCSEL ${ }^{[10]}$ etc.

Recently, a method based on an artificial neural network was proposed to classify mono-, and multi-modal
SM signals with success rate of $98.75 \%{ }^{[24]}$. However, this neural network based approach requires hand-crafted feature engineering. Pertinent features (based on temporal and spatial characteristics of SM fringes) are extracted only after performing correct SM fringe detection, a task which is difficult to achieve for noisy, experimental SM signals even when only one mode undergoes SMI, as attested by the use of advanced detection methods based on Hilbert transform [25], customized wavelet transform ${ }^{[26]}$, double-derivative ${ }^{[27]}$ and signal envelope tracking ${ }^{[28]}$ etc. However, in this Letter, multi-modality of the SM signal is identified without using robust fringe detection by evaluating four different SM signal statistics under different noise, optical feedback strength, amplitude of target vibration, and laser modality conditions. Use of majority vote among the four techniques has provided $100 \%$ identification success rate.

Various mono- and multi-modal SM signals were acquired by using two different LDs, L637P5 by Oclaro ${ }^{\circledR}$ and HL6501MG by Hitachi ${ }^{\circledR}$, one at a time. A polished metallic ring (mounted on a mechanical shaker, SF-9324 by $\mathrm{PASCO}^{\circledR}$ ) was used as the remote vibrating target. L637P5 LD has operating wavelength $\lambda_{o}$ of $637 \mathrm{~nm}$ and threshold current $I_{\text {th }}$ of $20 \mathrm{~mA}$, emitting $5 \mathrm{~mW}$ optical power. HL6501MG LD has $\lambda_{o}$ of $650 \mathrm{~nm}$ and $I_{\text {th }}$ of $45 \mathrm{~mA}$, providing $35 \mathrm{~mW}$ optical power. Each LD has a built-in photodiode through which SM signals were obtained. Different mono- and multi-modal SM signals were acquired under varying optical feedback and LD operating current $\left(I_{\text {oper }}\right)$ conditions. Multi-modal SM signals were observed to occur when both the optical feedback coupling (by using the focusing lens) and $I_{o p e r} I_{\text {th }}$ (by using higher $I_{o p e r}$ ) well exceeded unity. Fig. 1(a-b) presents two multi-modal SM signals based on HL6501MG LD with $I_{\text {oper }} / I_{\text {th }}$ ratio of $78 \mathrm{~mA} / 45 \mathrm{~mA}=1.73$, and $82 \mathrm{~mA} / 45 \mathrm{~mA}=1.82$ respectively under high optical feedback coupling. However, as optical feedback coupling was reduced (by de-focusing the lens) then mono-modal signal occurred even when $I_{o p e r} / I_{\text {th }}$ was 
(C) 2020 Optical Society of America]. One print or electronic copy may be made for personal use only. Systematic reproduction and distribution, duplication of

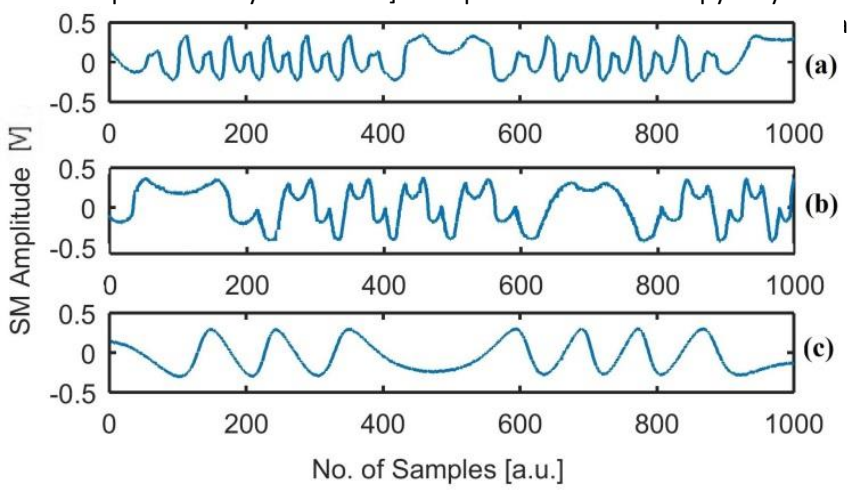

Fig. 1. Experimental multi- (a-b) and mono-modal (c) SM signals acquired under different optical feedback coupling and operating current conditions based on HL6501MG LD $\left(I_{t h}=45 \mathrm{~mA}\right)$ with $I_{\text {oper }}$ of (a-c) $78 \mathrm{~mA}$, and (b) $82 \mathrm{~mA}$.

1.73 (see Fig. 1 (c)). A dataset of $60 \mathrm{SM}$ signals (30 monoand 30 multi-modal SM signals) is used to verify the performance of the proposed techniques using SM signal statistical parameters.

Each of the proposed four different techniques for identification of SM multi-modality is detailed below.

Variance based technique (VBT) is based on the parameter varp-diff, which is a measure of peak to peak dynamic variation of an SM signal. Clearly, $\operatorname{var}_{p}$-diff should be generally greater for a multi-modal SM signal due to composition of different modes producing dissimilar multimodal fringes, as opposed to a mono-modal signal in which similar fringes occur (see Fig. 1). Consequently, larger variation in amplitude occurs in multi-modal signals as compared to mono-modal signals.

However, to perform VBT on normalized SM signal, two main phases are required: (1) customized local maxima detection and (2) estimation and analysis of varp-diff. Customized local maxima detection is done by the following steps, which are also presented in Fig. 2.

1) First, inter-maxima separation $\left(S M_{s e p}\right)$ is computed by using auto-correlation of SM signal. $S M_{\text {sep }}$ is indicative of the distance in between two consecutive maxima.

2) Then, the mean value of input SM signal, denoted by $\overline{S M}$, is computed.

3) Input SM signal (having $N$ number of samples) is divided into ' $n$ ' intervals by using $n=\operatorname{round}\left(N / S M_{\text {sep }}\right)$.

4) Then, local maxima of each interval is determined.

5) Valleys (SM signal portions with lower amplitude around local maxima) on left ( $l v)$ and right $(r v)$ is determined, for each local maximum of every interval.

6) Valley-less maxima are discarded and maxima with

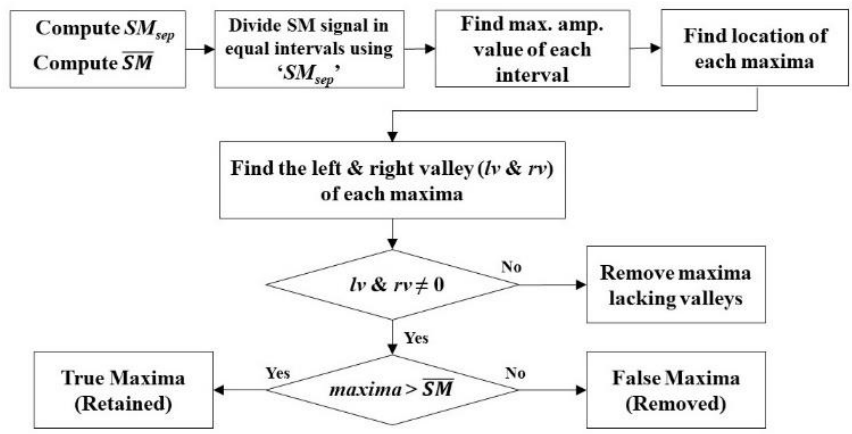

Fig.2.Flowchart of customized maxima detection technique http://dx.doi.org/10.3788/COL202018.011201

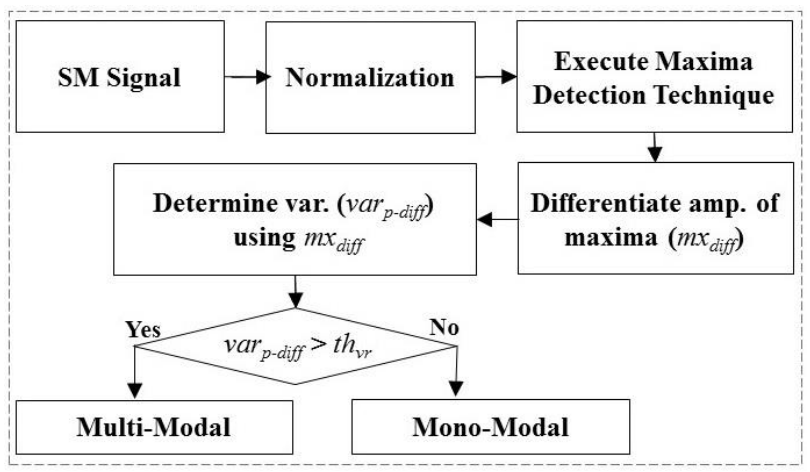

Fig. 3. Flowchart of variance based technique (VBT).

both valleys are retained.

7) Finally, amplitude values of maxima (having both valleys) are compared with $\overline{S M}$, and maxima with greater amplitude values are retained and considered as genuine maxima, while those with lower amplitude values are removed.

VBT second phase steps (see Fig. 3) are detailed below:

1) Differentiation of amplitude values of detected maxima $\left(m x_{\text {diff }}\right)$ is taken to determine_peak to peak dynamic variations ( $\left.\operatorname{var}_{p-\text { diff }}\right)$.

2) var $_{p-d i f f}$ is determined by taking variance of $m x_{\text {diff }}$ values.

3) A threshold value $\left(t h_{v r}\right)$ of $v^{2} p$-diff is employed and is compared with $\operatorname{var}_{p-\text { diff }}$ value of under-process SM signal to determine the modality of input signal. If $t_{\mathrm{vr}}<\operatorname{var}_{p-\text { diff, }}$ then input SM signal is considered a multimodal signal else it is considered a mono-modal signal. Note that this threshold (as well as subsequent thresholds in other techniques) is set in the light of various simulation results obtained under varying optical feedback coupling, amplitude of target vibration, and noise conditions as detailed ahead.

Kurtosis based technique (KBT) is based on the statistical parameter of kurtosis which is indicative of a signal's irregularity. Usually, amplitude of multi-modal SM signals is more irregular as compared to that of monomodal SM signals. Thus, kurtosis value of a SM signal, denoted by $S M_{k u r}$, can be used to extract information about its modality, where

$$
S M_{k u r}=\frac{\left.\sum_{i=1}^{N}\left(S M_{i}-\overline{S M}\right)^{4}\right) / N}{S^{4}}
$$

Here $S$ denotes the standard deviation value of the input SM signal. A threshold value $\left(t h_{k u r}\right)$ is set (by using simulation results) and is compared with $S M_{k u r}$. If $S M_{k u r}>$ $t h_{k u r}$ then input SM signal is considered multi-modal, else it is considered a mono-modal SM signal. Steps of KBT are shown in Fig (4).

Skewness based Technique (SBT) uses the statistical parameter of skewness which is a measure of asymmetry of the SM data around the sample mean. Conventionally, mono-modal SM signals are evenly distributed around the mean value. However, most commonly encountered multimodal signals are not even around the mean value. Thus, skewness parameter of a SM signal (denoted by $S M_{s k w}$ ) can also be useful in classifying the modality of a SM signal, 
(C) 2020 Optical Society of America]. One print or electronic copy may be made for personal use only. Systematic reproduction and distribution, duplication of

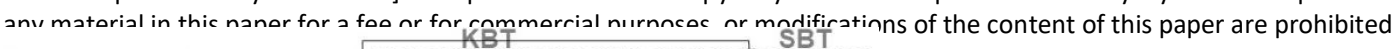

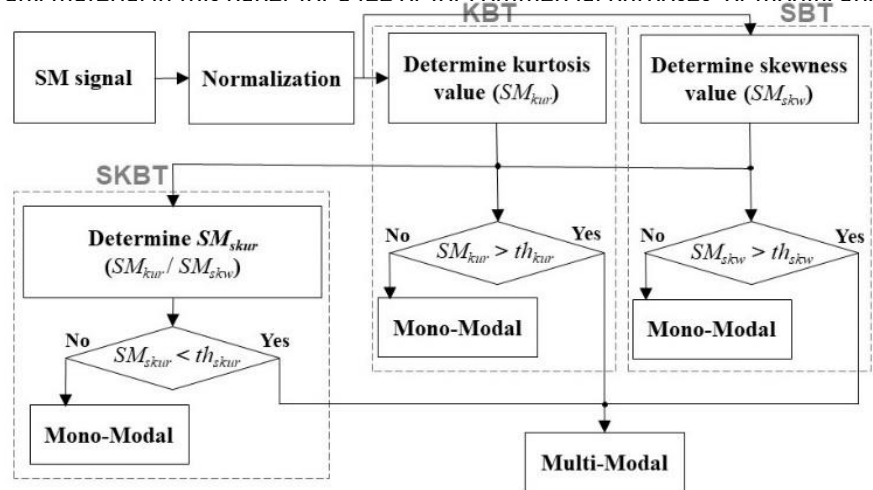

Fig. 4. Steps of Kurtosis based technique (KBT), skewness based technique (SBT), and skewness-kurtosis based technique (SKBT).

$$
S M_{S k w}=\frac{\left.\sum_{i=1}^{N}\left(S M_{i}-\overline{S M}\right)^{3}\right) / N}{S^{3}}
$$

Thus, $S M_{s k w}$ is determined, and is compared with the corresponding threshold value of skewness parameter $\left(t h_{s k w}\right)$ to ascertain the modality of input SM signal, Steps of this technique are also shown in Fig (4).

Skewness-kurtosis based technique (SKBT) is based on the ratio $\left(S M_{\text {skur }}=S M_{k u t} / S M_{s k w}\right)$ of above-mentioned SM signal parameters. As both $S M_{k u r}$ and $S M_{s k w}$ detailed above are good indicators of multi-modality, so their ratio ( $\left.S M_{\text {skur }}\right)$ is also investigated for identifying multi-modality. (Note that to avoid division by values of $S M_{s k w}$ approaching 0, all values of $S M_{s k w}<0.02$ were set to 0.02 to plot $S M_{\text {skur }}$ in Fig. $5(\mathrm{~d})$.) Absolute value of $S M_{\text {skur }}$ is compared with employed threshold value $\left(t h_{\text {skur }}\right)$. If $S M_{\text {skur }}<t h_{\text {skur }}$ then under-process SM signal is considered multi-modal else it is considered mono-modal. Steps of SKBT are also shown in Fig (4).

Let us now discuss how the various threshold values, used in each of the four presented techniques, were set by performing simulations for a representative sample of SM signals, by using SM model ${ }^{[14]}$ under different optical feedback coupling (such as frequently encountered weak-, and moderate-optical feedback regime ${ }^{[1,2]}$ ), amplitude of target vibration in terms of $\lambda_{o}$, and additive noise (resulting in different signal to noise ratio (SNR) of SM signals) conditions. Evolution of different parameters with respect to $C$, and amplitude of target vibration in the absence of noise for mono-model operation can be observed from Fig. 5. It can be observed from Fig. 5(a) that $v a_{p-d i f f}$ is always lower than 0.017 for mono-modal noiseless SM signals. In Fig. 5(b) $S M_{k u r}$ increases with $C$ which is expected since the more $C$ increases the more asymmetric the SM fringes become. Regarding $S M_{s k w}$ (see Fig. 5(c)), for low $C$ values (close to one), $S M_{s k w}$ is close to zero as positive and negative fringes are similar. Then, as $C$ increases, $\left|S M_{s k w}\right|$ value tends to increase due to the increasing asymmetry between the positive and negative fringes of SM signal.

Furthermore, to ascertain impact of additive noise on chosen parameters, simulations for weak feedback regime $(C=0.1)$ and moderate feedback regime $(C=4)$ are also performed (see Table 1 and Table 2 respectively). Two weak- and moderate-feedback regime SM signals under different noise conditions $(\mathrm{SNR}=10 \mathrm{~dB}$ and $\mathrm{SNR}=40 \mathrm{~dB})$ are graphically shown in Fig. 6 as well. Value of $C=4$ is
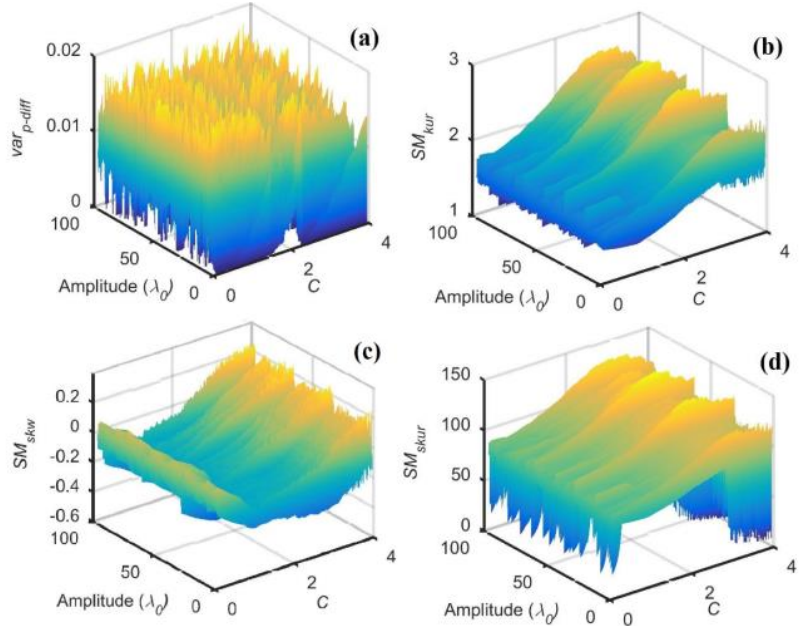

Fig. 5. Evolution of parameters with respect to $C$ and target vibration amplitude for noiseless mono-modal signal (a) $\operatorname{var}_{p-\text { diff }}$ (b) $S M_{k u r}$, (c) $S M_{s k w}$, and (d) $\left|S M_{s k u r}\right|$

specifically chosen to perform noise analysis as it generally corresponds to the worst-case statistical parameter values.

It can be observed from Table 1 and Fig. 6 that value of parameters such as var $_{p-d i f f}$ is decreasing significantly as SNR improves. Higher SNR values result in fewer local maxima generated by noise (and thus not genuine fringes) to be wrongly considered as fringe. Therefore, the calculation of $\operatorname{var}_{p-\text { diff }}$ will not take them into account and

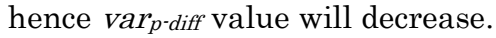

Conduct of these simulations under different levels of noise, amplitude of target vibration, and OF coupling provides information about the expected range and worstcase value of proposed parameters, resulting in extraction of different threshold values (see Table 3).

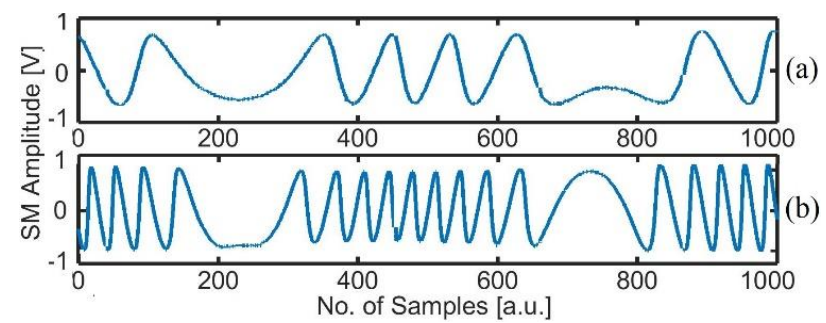

Fig. 7. (a-b) Sample correctly identified experimental mono-modal SM signals.

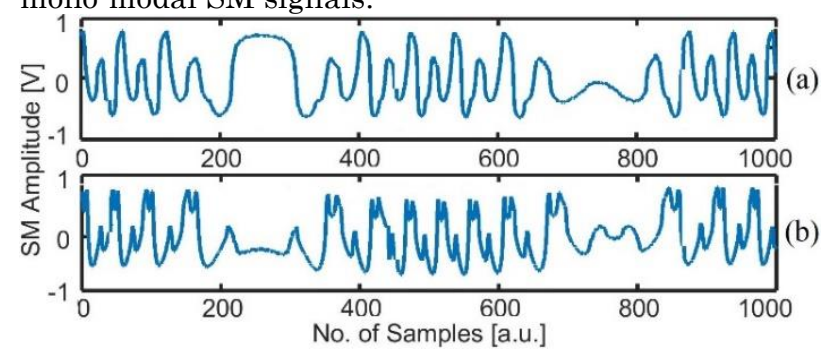

Fig. 8. Sample correctly identified experimental (a) bimodal-, and (b) tri-modal- SM signals. 
(C) 2020 Optical Society of America]. One print or electronic copy may be made for personal use only. Systematic reproduction and distribution, duplication of any material in this paper for a fee or for commercial purposes, or modifications of the content of this paper are prohibited

Table 1. Values of statistical parameters of simulated normalized mono-modal SM signals for varying SNR under $\underline{\text { weak-feedback regime for } C=0.1 \text {, and amplitude }=5 \lambda_{\circ}}$.

\begin{tabular}{cccccc} 
Techs. & Feats. & $\begin{array}{c}\text { SNR } \\
(10 \mathrm{~dB})\end{array}$ & $\begin{array}{c}\text { SNR } \\
(20 \mathrm{~dB})\end{array}$ & $\begin{array}{c}\text { SNR } \\
(30 \mathrm{~dB})\end{array}$ & $\begin{array}{c}\text { SNR } \\
(40 \mathrm{~dB})\end{array}$ \\
\hline VBT & var $_{\text {-diff }}$ & 0.015 & 0.012 & 0.012 & 0.008 \\
KBT & $S M_{\text {kur }}$ & 1.718 & 1.452 & 1.433 & 1.429 \\
SBT & $S M_{\text {skw }}$ & 0.149 & 0.144 & 0.142 & 0.141 \\
SKBT & $\left|S M_{\text {skur }}\right|$ & 11.458 & 10.053 & 9.821 & 9.510 \\
\hline
\end{tabular}

Table 2. Statistical parameters' values for simulated normalized mono-modal SM signals for varying SNR under moderate-feedback regime $(C=4)$, and amplitude $=5 \lambda_{0} \cdot(\mathrm{a})$

\begin{tabular}{cccccc} 
Techs. & Feats. & $\begin{array}{c}\text { SNR } \\
(10 \mathrm{~dB})\end{array}$ & $\begin{array}{c}\text { SNR } \\
(20 \mathrm{~dB})\end{array}$ & $\begin{array}{c}\text { SNR } \\
(30 \mathrm{~dB})\end{array}$ & $\begin{array}{c}\text { SNR } \\
(40 \mathrm{~dB})\end{array}$ \\
\hline VBT & var $_{p \text { diff }}$ & 0.016 & 0.011 & 0.007 & 0.006 \\
$\mathrm{KBT}$ & $S M_{k u r}$ & 2.267 & 2.253 & 2.099 & 2.007 \\
SBT & $S M_{\text {skw }}$ & -0.345 & -0.340 & -0.326 & -0.320 \\
SKBT & $\left|S M_{\text {skur }}\right|$ & 6.777 & 6.627 & 6.162 & 6.103 \\
\hline
\end{tabular}

Table 3. Extracted threshold values of proposed statistical parameters based on simulations on mono-modal SM signals under varying optical feedback, vibratän amplitude, and signal to noise ratio

\begin{tabular}{ccccc}
\hline & VBT & KBT & SBT & SKBT \\
\hline Features & var $_{p \text {-diff }}$ & $S M_{\text {kur }}$ & $S M_{\text {skw }}$ & $\left|S M_{\text {skur }}\right|$ \\
Threshold values & 0.017 & 2.7 & 0.2 & 5 \\
\hline
\end{tabular}

In order to determine the performance of proposed techniques, experimental dataset was tested to identify the modality of these SM signals by using threshold values of Table 3 . Results are presented in Table 4 , where $N_{\text {mon }}$ and $N_{m u l}$ indicates number of tested mono-, and multi- modal SM signals respectively. Likewise, $N_{m o n-T i}$ and $N_{m u l-T i}$ indicates number of truly identified mono- and multimodal SM signals respectively. Furthermore, $N_{\text {mon-Fi }}$ and $N_{m u l-F i}$ are the number of SM signals which are falsely
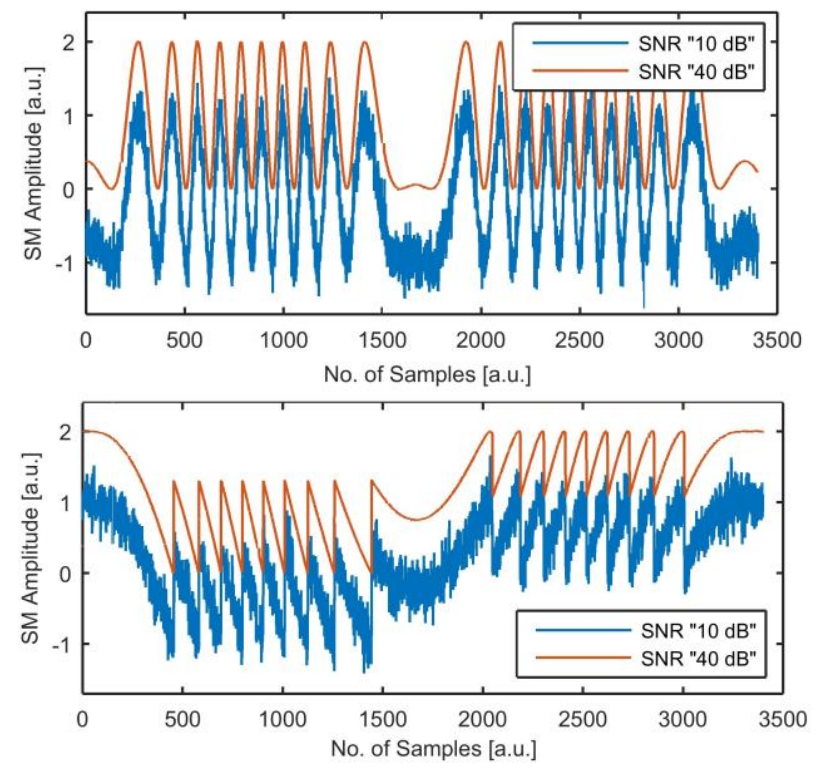

Fig. 6. Simulated mono-modal SM signals with SNR of 10 $\mathrm{dB}$ and $40 \mathrm{~dB}$ in case of (a) weak feedback regime $(C=$ $0.1)$ and (b) moderate feedback regime $(C=4)$. identified as mono-, and multi-modal SM signals respectively. $N_{t o-T i}$ represents the total number of truly identified SM signals. In the last column, $R_{S}$ represents the overall success rate of proposed techniques.

Table 4 Performance of proposed techniques by testing experimentally acquired dataset of $60 \mathrm{SM}$ signals

\begin{tabular}{cccccccc}
\hline Techs. & $\begin{array}{c}N_{\text {mon }} \\
\boldsymbol{N}_{\text {mul }}\end{array}$ & $N_{\text {mon_Ti }}$ & $N_{\text {mul_Ti }}$ & $N_{\text {mon Fi }}$ & $N_{\text {mul Fi }}$ & $N_{\text {to-Ti }}$ & $R_{S}$ \\
\hline VBT & $30 / 30$ & 30 & 21 & 9 & 0 & 51 & 85 \\
KBT & $30 / 30$ & 24 & 21 & 9 & 6 & 45 & 75 \\
SBT & $30 / 30$ & 29 & 26 & 4 & 1 & 55 & 91 \\
SKBT & $30 / 30$ & 26 & 30 & 0 & 4 & 56 & 93 \\
MV & $30 / 30$ & 30 & 30 & 0 & 0 & 100 & 100 \\
\hline
\end{tabular}

An analysis of misidentified signals led to the observation that misidentification by the proposed techniques occurred for different SM signals. So, majority voting (MV) based on results of the four techniques was undertaken (for each tested signal) resulting in 100\% success rate. If lower number of parameters is used for the sake of reducing the complexity of the blind identification then $R_{s}=95 \%$ if VBT is not used while $R_{s}=92 \%$ if only VBT and SBT are used, inclusive of MV in both the cases. Some correctly identified mono- and multi-modal experimental SM signals are graphically presented in Fig. 7, and Fig. 8, respectively.

To conclude, an OF based LD can provide multi-modal SM signal in place of usually encountered mono-modal SM signal because of mode-hopping caused by change in operating conditions such as LD-to-target distance. This can cause misinterpretation of SM fringe-count, resulting in drastic increase in metric measurement error. To avoid this error, a continuous monitoring of SM signal is necessary, so that, as SM signal becomes multi-modal, it could be detected immediately and possibly reverted back to mono-modal behavior (e.g. by changing LD current or OF strength). In this Letter, different techniques, based on SM signal statistics, are evaluated for future continuous monitoring of emission modality of low-cost LD based SM sensor. These proposed techniques have been successfully tested on experimentally acquired mono-, and multi-modal SM signals with success rate of $85 \%$ (VBT), $75 \%$ (KBT), 91\% (SBT), and 93\% (SKBT). Importantly, use of majority voting among the four proposed techniques has provided $100 \%$ success rate of SM modality identification.

\section{References}

1. Taimre T, Nikolić M, Bertling K, Lim YL, Bosch T, Rakic AD. Laser feedback interferometry: A tutorial on the self-mixing effect for coherent sensing. Advances in Optics and Photonics. 2015;7(3):570-631.

2. Donati S. Developing self-mixing interferometry for instrumentation and measurements. Laser \& Photonics Reviews. 2012;6(3):393-417.

3. Sun W, Liu J, Gui H, Lu A, Wang H, Lu Y. Simultaneous measurement of vibration amplitude and rotation angle based on a single-channel laser selfmixing interferometer. Chinese Optics Letters. 2016;14(2):021201. 
(C) 2020 Optical Society of America]. One print or electronic copy may be made for personal use only. Systematic reproduction and distribution, duplication of any material in this paper for a fee or for commercial purposes, or modifications of the content of this paper are prohibited

4. Wang S, Wang X. Resonant characteristics of microcantilever by using self-mixing interferometer based on phase reconstruction method. Chinese Optics Letters. 2010;8(2):177-180.

5. Wang H, Shen J. Size measurement of nano-particles using self-mixing effect. Chinese Optics Letters. 2008;6(11):871-874.

6. Wang $\mathrm{H}, \mathrm{Zhao} \mathrm{T}, \mathrm{Xu}$ J, et al. Optimized design of laser range finding system using the self-mixing effect in a single-mode VCSEL. Chinese Optics Letters. 2006;4(2):87-90.

7. Sun X, Wang H, Liu B, Yu Y. Measurement of microsurface topography using a self-mixing optical configuration. Optical Engineering. 2018;57(5):051503.

8. Wu Z, Duan N, Wang C, Li J, Zhu J. Experimental study on acoustic-to-seismic landmine detection based on laser self-mixing interferometer. Paper presented at: Sixth International Conference on Optical and Photonic Engineering (icOPEN 2018)2018.

9. Ruiz-Llata M, Lamela H. Self-mixing technique for vibration measurements in a laser diode with multiple modes created by optical feedback. Applied Optics. 2009;48(15):2915-2923.

10. Tucker JR, Rakić AD, O'Brien CJ, Zvyagin AV. Effect of multiple transverse modes in self-mixing sensors based on vertical-cavity surface-emitting lasers. Applied Optics. 2007;46(4):611-619.

11. Zabit U, Shaheen K, Naveed M, Bernal OD, Bosch T. Automatic Detection of Multi-modality in Self-Mixing Interferometer. IEEE Sensors Journal. 2018;18(22):9195-9202..

12. Pham T, Seat H, Bernal O, Surre F, Bosch T. Selfmixing sensing under strong feedback using multimode semiconductor lasers. Paper presented at: Lasers and Electro-Optics Pacific Rim (CLEO-PR), 2013 Conference on 2013.

13. Bernal OD, Zabit U, Bosch TM. Robust method of stabilization of optical feedback regime by using adaptive optics for a self-mixing micro-interferometer laser displacement sensor. IEEE Journal of Selected Topics in Quantum Electronics. 2015;21(4):336-343.

14. Kliese R, Taimre T, Bakar AAA, et al. Solving selfmixing equations for arbitrary feedback levels: a concise algorithm. Applied Optics. 2014;53(17):37233736.

15. Bernal OD, Zabit U, Bosch T. Classification of laser self-mixing interferometric signal under moderate feedback. Applied Optics. 2014;53(4):702-708.

16. Gao Y, Yu Y, Xi J, Guo Q, Tong J, Tong S. Improved method for estimation of multiple parameters in selfmixing interferometry. Applied Optics. 2015;54(10):2703-2709.

17. Ehtesham A, Zabit U, Bernal O, Raja G, Bosch T. Analysis and Implementation of a Direct Phase Unwrapping Method for Displacement Measurement using Self-Mixing Interferometry. IEEE Sensors Journal. 2017;17(22):7425-7432.

18. Fan Y, Yu Y, Xi J, Chicharo JF. Improving the measurement performance for a self-mixing interferometry-based displacement sensing system. Applied Optics. 2011;50(26):5064-5072.

19. Khan ZA, Zabit U, Bernal OD, Ullah MO, Bosch T. Adaptive cancellation of parasitic vibrations affecting a self-mixing interferometric laser sensor. IEEE Transactions on Instrumentation and Measurement. 2017;66(2):332-339.

20. Huang Z, Li C, Sun X. Piece-wise transition detection algorithm for a self-mixing displacement sensor. Chinese Optics Letters. 2013;11(9):091203.

21. Lv L, Gui H, Xie J, et al. Effect of external cavity length on self-mixing signals in a multilongitudinal-mode Fabry Perot laser diode. Applied Optics. 2005;44(4):568571.

22. Zhao Y, Zhou J, Lu L. Measurement of the Free Spectral Range of the laser cavity based on multilongitudinal mode Laser Self-mixing Vibrator. Measurement. 2018.

23. Keeley J, Freeman J, Bertling K, et al. Measurement of the emission spectrum of a semiconductor laser using laser-feedback interferometry. Scientific Reports. 2017;7(1):7236.

24. Zabit U, Shaheen K, Naveed M, Bernal OD, Bosch T. Automatic Detection of Multi-Modality in Self-Mixing Interferometer. IEEE Sensors Journal. 2018;18(22):9195-9202.

25. Arriaga AL, Bony F, Bosch T. Real-time algorithm for versatile displacement sensors based on self-mixing interferometry. IEEE Sensors Journal. 2016;16(1):195202.

26. Bernal OD, Seat HC, Zabit U, Surre F, Bosch T. Robust detection of non-regular interferometric fringes from a self-mixing displacement sensor using Bi-Wavelet transform. IEEE Sensors Journal. 2016;16(22):79037910 .

27. Magnani A, Pesatori A, Norgia M. Self-mixing vibrometer with real-time digital signal elaboration. Applied Optics. 2012;51(21):5318-5325.

28. Siddiqui AA, Zabit U, Bernal OD, Raja G, Bosch T. All analog processing of speckle affected self-mixing interferometric signals. IEEE SenS J. 2017;17(18):58925899. 